\title{
Análisis de los criterios para la valoración turística del patrimonio natural
}

\author{
María José Viñals Blasco \\ Universidad Politécnica de Valencia \\ mvinals@cgf.upv.es \\ Maryland Morant González \\ Universidad Politécnica de Valencia \\ marmogon@cgf.upv.es \\ Rubén Quintana \\ Universidad de Buenos Aires \\ rubenq@ege.fcen.uba.ar
}

\section{RESUMEN}

La puesta en valor turístico de espacios sensibles comporta una cuidadosa planificación y gestión que debe basarse en la utilización de herramientas técnicas y en criterios de base científica. En este trabajo se analiza la oportunidad de emplear algunos criterios que han sido seleccionados y adaptados para ser utilizados para la toma de decisiones en materia de gestión turística del patrimonio natural. Así, en primera instancia y para realizar la valoración intrínseca se proponen: significancia, representatividad, singularidad, naturalidad, e integridad. Para la valoración recreativa se estudian: fragilidad, atractividad, accesibilidad, disponibilidad en el tiempo y en el espacio, factibilidad y potencial para la educación y la interpretación del patrimonio. Las conclusiones del estudio muestran los beneficios de utilizar métodos rigurosos y científicos de trabajo para la planificación y gestión de destinos turísticos de alto valor ecológico por cuanto que se pueden aplicar medidas preventivas previamente a la puesta en marcha de actividades recreativas y de sus posibles impactos dado que éstas siempre son más recomendables que tener que aplicar medidas correctoras.

Palabras clave: Actividades Recreativas y turísticas, Valoración Recreativa del Patrimonio Natural 


\title{
Analysis the assessment criteria touristic natural heritage
}

\author{
María José Viñals Blasco \\ Polytechnic University of Valencia \\ mvinals@cgf.upv.es
}

Maryland Morant González

Polytechnic University of Valencia

marmogon@cgf.upv.es

Rubén Quintana

University of Buenos Aires

rubenq@ege.fcen.uba.ar

\begin{abstract}
Mobilizing sensitive resources for touristic purposes compromises an accurate planning and management that must be based on the use of technical tools and scientific criteria. This paper analyses the opportunity to implement a selection of criteria for the Resources Recreational Assessment in the decision making process in relation to tourism management. Therefore, the first step is to evaluate the Intrinsic Value of the resources by using criteria such as significance, representativeness, singularity, wilderness and integrity. For an specific recreational evaluation fragility, attractiveness, accessibility, availability in time and space, feasibility and potential for education and heritage interpretation have been proposed. The outcomes show the benefits that a rigorous and scientific method of work for tourist planning in tourist destinations with high ecological values can bring in order to apply preventive measures before recreational activities commence and can anticipate various possible impacts, given that, these are more recommendable than corrective measures.
\end{abstract}

Keywords: Recreational and tourist activities, Recreational Assessment of Natural Heritage 


\section{INTRODUCCIÓN}

La experiencia muestra que las actividades recreativas no planificadas traen a menudo aparejadas consecuencias no deseadas para los ecosistemas donde se escenifican, acarreando pérdida de bienes y servicios ambientales indispensables para el desarrollo y el mantenimiento de las sociedades humanas. Incluso en el caso de lugares remotos que, por sus características ambientales, se encuentran poco poblados o localizados en regiones poco accesibles, encontramos huellas de la actividad humana, difíciles de erradicar. Por esa razón, cualquier actividad recreativa que vaya a llevarse a cabo debe ser cuidadosamente planificada y gestionada en base a la utilización de criterios científicos y herramientas técnicas adecuadas para poder decidir la mejor localización para la actividad, dónde los impactos sobre los recursos sean mínimos.

Un elemento importante a tener en cuenta es que los impactos causados por la recreación están estrechamente relacionados con el tipo de actividad que se vaya a desarrollar; así variará si se trata de una actividad lúdica, interpretativo-educativa, y/o deportivo aventurera, (Viñals, 1999). Por otra parte, el modelo turístico aplicado, la decisión de dónde, cuándo y cómo implantar dicha actividad turística se relaciona también con la vocación del territorio (aptitud e idoneidad) y con la fragilidad y vulnerabilidad de sus recursos (Viñals, 2002) que son los factores limitantes más importantes para el desarrollo de cualquier actividad.

El presente trabajo analiza la oportunidad de utilizar una serie de criterios científicos para la valoración recreativa de los recursos naturales, en aras de facilitar la toma de decisiones en cuanto a poner en marcha una actividad recreativa sobre un ecosistema sensible. La valoración recreativa de los recursos es una herramienta de base científica basada en la utilización de unos criterios específicos que permiten la evaluación de los recursos en base a su atractivo turístico. Este sistema de valoración recreativa ha sido empleado de diversos casos de estudio que el grupo de investigación ha llevado a cabo donde había en juego recursos frágiles como era el caso de los humedales de la cuenca mediterránea (Viñals et. al, 2005) o en yacimientos arqueológicos, como el poblado ibérico del Castellet de Bernabé en Llíria (Valencia). Ello nos ha permitido mejorar el método de trabajo y refinar las herramientas.

Los instrumentos iniciales de los cuales parte la valoración recreativa son los inventarios, que facilitan la adquisición ordenada y sistematizada de información sobre los recursos existentes en la zona que potencialmente podrían constituir el elenco de atractivos del lugar.

Existen muchas propuestas metodológicas de cómo recoger, almacenar, ordenar y clasificar la información asociada a los recursos tanto naturales como culturales o de otro tipo. De hecho desde la década de los años '60 del siglo XX en que se produce el "boom turístico" comienzan a prodigarse metodologías para ello como la de Defert (1960), siendo la de la Organización de Estados Americanos (1978) la más utilizada por autores dedicados a estos temas. Posteriormente, la Organización Mundial del Turismo (1978) incorpora los inventarios 
como uno de los instrumentos de trabajo básicos y autores como Burkart y Medlik (1986), Gunn (1988), Backman et al. (1991), Leno (1993), Nusser y Goebel (1997) se adentran en el tema con el ánimo de enfatizar las bondades de su utilización en los estudios turísticos. Los inventarios deben ser, en definitiva, documentos que recojan información como mínimo sobre la localización del recurso, tipología y caracterización de los mismos, cronología, usos actuales e importancia económica y social que la sociedad les atribuye, el tipo de gestión de que es objeto el recurso, valoración de su estado de conservación, riesgos y amenazas de que es objeto, y además toda la documentación gráfica y cartográfica necesaria.

Hay que mencionar que muchos estudios turísticos concluyen con la fase de inventario, hecho claramente insuficiente para la toma de decisiones de planificación y gestión turística del territorio. De otro lado, hay que mencionar que estos inventarios deben estar sometidos siempre a procesos de actualización ya que de otra manera no resultan útiles.

La valoración de los recursos se inicia una vez se han inventariado convenientemente. En primer lugar, hay que efectuar una valoración intrínseca de los mismos para hacernos una idea de su valor como tal recurso y, en segundo lugar, se llevará a cabo una valoración recreativa de los mismos. A la conclusión de las mismas, se tendrá una idea aproximada sobre la aptitud e idoneidad de los recursos para poder ser utilizados en actividades recreativas y se podrá entonces comenzar la fase de planificación.

Hay que mencionar que en relación a las valoraciones de los recursos, la Ecología ha sido la ciencia que ha dedicado mayores esfuerzos para el establecimiento de criterios para la toma de decisiones, fundamentalmente ante la necesidad de establecer programas de conservación y protección de espacios y especies o proyectos de restauración (Mallarach, 1999). Este trabajo, puesto que se centra en el estudio de recursos naturales, incorpora criterios de valoración intrínseca de corte ecológico. Los estudios metodológicos de valoración recreativa de los recursos son muy escasos y el establecimiento justificado de unos criterios de evaluación aún más. Para este cometido, el trabajo ha partido de la utilización de criterios de base ecológica, junto con otros del ámbito de las Ciencias Sociales. Estos criterios han tenido que ser adaptados para poder hacer extensiva su utilización a todo tipo de recursos (humedales, monumentos, restos arqueológicos, paisajes culturales, ornitofauna, etc.).

\section{LA VALORACIÓN INTRÍNSECA DE LOS RECURSOS}

Esta evaluación consiste en determinar el valor o atributos propios que en sí mismos detentan los elementos del ecosistema que se analiza en relación a su pertenencia a tal grupo específico (valor arqueológico si es una ruina, valor ecológico si es una especie, valor geológico si es un yacimiento fosilífero, etc.). Se contemplan como criterios de valoración en esta fase: significancia, representatividad, singularidad, rareza y naturalidad e integridad. Todo ello aplicado a recursos de tipo geológico, geomorfológico, faunístico, florístico, ecológico, hidrológico y paleontológico, entre otros. Este último criterio, en el caso de patrimonio cultural se asimila a la autenticidad.

La significancia se refiere al valor o importancia del elemento natural, sean especies de fauna o flora así como los elementos abióticos, en función de su relación con el entorno y a las 
características propias que presenta en función de la clase o tipo en la que haya sido incluido (valor naturalístico, valor científico, valor social universal, etc.). Un ejemplo claro en este sentido es el de los primates superiores, a los que siempre se les atribuye una especial significancia desde todas las perspectivas, por sus estrechos vínculos con la especie humana. Otro caso para ilustrar este concepto nos lo procuran las plantas con propiedades curativas y medicinales, a las cuales se les considera también de gran importancia por las múltiples aplicaciones que de ellas se derivan.

La representatividad hace mención al grado en que el elemento natural en cuestión presenta las características o atributos propios del grupo al que pertenece. Este criterio puede entenderse desde dos perspectivas: la representatividad típica (cuando un rasgo del lugar o la comunidad es común; e.g., Phoenicopterus ruber que es un flamenco que habita normalmente en los humedales salinos) o puede representar un rasgo singular o único del lugar o comunidad (característica excepcional minoritaria). Por ejemplo, se puede decir que las albuferas y marjales del levante español son representativas de los humedales de esta región porque presentan la característica de tener aguas dulces lo que las distingue del resto de otros humedales ribereños del mediterráneo.

La singularidad se establece en función de la rareza que el elemento presenta en relación con las características propias de la clase o tipo a la que pertenece. Este criterio se define considerando la escala geográfica de distribución del elemento y el número de elementos. Así, puede tener dos aproximaciones fundamentales, la referida a singularidad frente al entorno más o menos inmediato (nivel local) y la referida a su excepcionalidad a grandes escalas regional o continental (Cadiñanos y Meaza 1998). Un ejemplo de rareza natural a nivel local y regional es la Sequoia gigante (Sequoia gigantea) cuyos mejores ejemplares los encontramos en el Parque Nacional de este nombre en California (EE.UU.), la cual era rara aún antes de que los humanos entraran en escena.

Por otra parte, Rabinowitz (1981) estima que los recursos abióticos pueden considerarse raros por su singularidad morfológica y/o por la excepcionalidad de los procesos que dieron origen a los mismos. Así, desde una aproximación a gran escala, monolitos, arcos o rocas superpuestas son elementos singulares del paisaje resultado de procesos de erosión diferencial sobre determinados tipos de roquedo. Es el caso del arco sobre arenisca del Arches Nacional Monument en Utah (EE.UU.) o los cerros testigos de arenisca compacta del Monument Valley en Arizona (EE.UU.), o siguiendo con las formaciones de arenisca, las que encontramos en el Parque Arqueológico de Petra (Jordania). Otros ejemplos de formaciones excepcionales a nivel mundial, lo constituyen dos de los destinos turísticos más atractivos del planeta: Pamukale (Turquía) y Mammoth Hot Springs (Parque Nacional de Yellowstone, EE.UU.) resultado de la combinación de surgencias termales ricas en minerales calcáreos.

Asociados al concepto de rareza aparecen, ligados a los recursos bióticos, el de endemicidad y relictismo, referido a aquellos taxones o asociaciones propios y exclusivos de un determinado espacio biogeográfico o representativos de otra época y otras condiciones ambientales que perduran con escasa y muy localizada representación. Las islas oceánicas suelen ser ejemplos típicos de sitios con alto grado de endemismos debido fundamentalmente al prolongado aislamiento geográfico que éstas han sufrido a lo largo de su historia geológica. 
No hay que descartar que parte del atractivo turístico de las islas resida en su carácter único ligado posiblemente a este factor. Un ejemplo paradigmático lo constituye el archipiélago de las Islas Galápagos (Ecuador), donde de las más de 2.900 especies marinas descritas, el 18,2\% es endémica al igual que ocurre con la fauna terrestre, entre la que destacamos la tortuga gigante (Geochelone elephantopus) atractivo fundamental para los visitantes que llegan hasta este espacio. En el archipiélago de Juan Fernández, recientemente afectado por los sismos que tuvieron lugar en Chile, cerca del $80 \%$ de la flora de la isla Robinson Crusoe o Más a Tierra es endémica. Con respecto a áreas continentales, hay que destacar que España es uno de los países europeos con más endemismos (alrededor de 1.500 en la península más unos 500 en las Islas Canarias). La presencia de elementos endémicos o relictuales le otorgaría al sitio una situación de gran fragilidad respecto a otros espacios puesto que al ser lugares con presencia única de ciertos recursos, su degradación o destrucción podría llevar a la desaparición de los mismos. Los endemismos suelen ser por ello muy vulnerables a la transformación de sus ecosistemas, ya que sus hábitats se encuentran en regiones donde ciertas poblaciones evolucionaron en aislamiento $\mathrm{y}$ en las cuales las especies desarrollaron características específicas relacionadas con su entorno.

La naturalidad es un concepto que se opone a la artificialidad del lugar y por tanto tiene que ver con el buen estado de conservación del ecosistema. La artificialidad evalúa el grado de transformación de un espacio natural. Es un indicador de lo alejado que está de su estado prístino. Se considera que cuanto más artificializado esté el espacio natural, mayor va a ser la fragilidad de las áreas naturales remanentes, ya que deberán soportar presiones cada vez mayores. Esta idea va muy ligada también al concepto de integridad ecológica que se define como la habilidad de mantener equilibrado el conjunto de todos los elementos abióticos (agua, rocas, etc.) y bióticos (especies y comunidades biológicas) que constituyen el ecosistema y los procesos que allí tienen lugar (Karr, 1996). La integridad de un ecosistema hace que esté presente cierto nivel de resiliencia (dependiendo del tipo de ecosistema que se trate) ante los disturbios ambientales (Strickland-Munro et al., 2010). Aún así, la proximidad a fuentes de impacto humano constituye siempre un gran riesgo para el mantenimiento del carácter natural de un sitio.

Relacionado con el concepto de integridad se encuentra el de biodiversidad o diversidad biológica, que tiene una gran relevancia en la valoración intrínseca de los ecosistemas. Por diversidad biológica o biodiversidad se entiende la variedad de organismos vivientes considerados a todos los niveles de organización, incluyendo diversidad a nivel genético, de especies y de otros niveles taxonómicos superiores y a la variedad de hábitats y ecosistemas así como a los procesos que tienen lugar en ellos (Meffe y Carroll, 1997). El mantenimiento de la diversidad biológica de un sistema natural asegura el mantenimiento de su integridad $y$, por ende, de las funciones ecosistémicas que dicho sistema posee. A medida que se incrementa la biodiversidad, aumenta la probabilidad de que un ecosistema sea más estable y más resiliente (Naeem, 1998). Por otra parte, la diversidad geológica, geomorfológica, edáfica, hídrica y climática, son la base del nivel de heterogeneidad ambiental presente en un territorio $y$, por lo tanto, del número de especies que un sistema puede sustentar. 
A propósito de la biodiversidad, hay que mencionar que los humedales tienen en este criterio su más importante pilar para fomentar su conservación y protección. En cambio, hay que hacer notar que la biodiversidad no siempre resulta atractiva desde el punto de vista turístico. Está siempre en función de las especies que compongan esa biodiversidad. Así, retomando el ejemplo anterior de los humedales, pese a ser uno de los ecosistemas más biodiversos del mundo no son los más atractivos para el público porque las especies que lo componen son catalogadas como fauna molesta (insectos, reptiles, etc.) y únicamente las aves escapan a esta consideración y resultan atractivas a los ojos del visitante generalista. Otros ecosistemas menos biodiversos pero con alguna especie emblemática como son los bosques de bambú con osos panda, resultan más atractivos al público.

\section{CRITERIOS DE VALORACIÓN RECREATIVA}

El proceso de valoración recreativa de los recursos se lleva a cabo mediante la utilización de otros criterios. A partir de los resultados de este análisis es factible dilucidar la aptitud e idoneidad que tienen los recursos como atractivos para el público o para acoger determinadas actividades. Un criterio fundamental y prioritario en este análisis es el de fragilidad, ya que resulta un indicador de la resistencia que el ecosistema o recurso en sí va a presentar frente a los posibles impactos que puedan derivarse de la actividad recreativa y de los propios visitantes. Un paso posterior es el análisis de los criterios de: atractividad así como de su accesibilidad, su disponibilidad en el tiempo y en el espacio y su aptitud potencial para la educación y la interpretación y la factibilidad de utilizarlo para tal fin.

La fragilidad se relaciona con la posibilidad de destrucción y/o degradación del recurso o de un sistema en función de su propia dinámica ecológica y evolutiva y existen muchos factores relacionados con el análisis de la fragilidad de un recurso o un ecosistema. Es lo opuesto al criterio de resistencia, que es la habilidad de un ecosistema para resistir a un determinado impacto (De Angelis et al., 1989).

El criterio de endemicidad y rareza, ya tratados, habitualmente van coaligados a éste. Otro factor relacionado es el de amenaza de extinción. Un recurso amenazado es aquel que se encuentra en regresión y en peligro más o menos inmediato de desaparición como resultado, básicamente, de la acción humana. Se considera que la presencia de recursos amenazados puede afectar la fragilidad de un ecosistema debido a que se pierden especies o recursos (como los distintos tipos de hábitats) que afectan la integridad del mismo.

Para el caso de recursos bióticos, la referencia más generalizada es la de categorías de estatus de conservación de especies que se establecen en el libro rojo de la UICN (2001) ${ }^{1}$. Un conocido ejemplo de especie en peligro de extinción en España lo constituye el lince ibérico (Lynx pardinus), catalogado como el último gran gato salvaje europeo. Otro ejemplo son las playas mediterráneas, ya que constituyen un caso de recurso abiótico amenazado. Este tipo de ecosistema es el soporte de una de las modalidades turísticas más extendidas como es el

\footnotetext{
${ }^{1}$ La IUCN ha compilado listas de especies en dichas categorías y la inclusión de éstas en cada una se ha basado en una serie de consideraciones que se encuentran descritas en "Guidelines on the application of the IUCN Red List Criteria at national or regional levels"; http://www.iucn.org/themes/ssc/redlists/regionalguidelines.htm).
} 
"turismo de sol y playa". Estos ecosistemas se encuentran en franco retroceso por la acción combinada de factores muy diversos como el ascenso del nivel marino resultado del cambio climático, el desequilibrio creado por la introducción de estructuras que interrumpen los flujos sedimentarios longitudinales en el litoral, la falta de aportes sedimentarios fluviales que nutren el sistema y por el uso turístico generalizado de los mismos. El problema resulta que restaurar la forma de una playa es una tarea relativamente sencilla, pero restaurar un proceso dinámico de alimentación sedimentaria costera resulta mucho más complejo, a veces se trata de un impacto irreversible.

El criterio de fragilidad va muy ligado también al de vulnerabilidad, que se puede definir como la falta de capacidad para soportar determinados impactos causados por agentes ajenos a su propia dinámica natural, especialmente de origen humano (Cadiñanos y Meaza, 1998). Existe un estudio muy útil de Liddle (1997) para este tipo de valoraciones, dedicado a analizar la vulnerabilidad de especies de flora y fauna frente a los diferentes impactos derivados de la recreación.

Así pues, podemos ver que el estudio de la fragilidad de los ecosistemas es fundamental para conocer las oportunidades para el uso público. De esta forma, se establece una relación inversamente proporcional entre fragilidad y desarrollo de actividades recreativas: a mayor fragilidad menos posibilidades de utilizar los recursos con fines recreativos.

Por otra parte, existe un grupo de criterios que se relacionan con la atracción y el interés que un recurso despierta sobre el público. El más importante en este sentido es la atractividad que es un criterio basado en parámetros de tipo estético, emotivo y perceptual. Así, se valora la belleza, originalidad, el simbolismo y las emociones que provoca el recurso en las personas. Como se puede observar, se trata de un criterio para la valoración recreativa, con una gran componente de subjetividad marcada por unas preferencias que dependen de las características de los visitantes (edad, cultura, educación, nacionalidad, etc.). De cualquier manera, hay elementos objetivos para la valoración como son: la unicidad, el buen estado de conservación, la calidad escénica del entorno y el tamaño o superficie del sitio.

La unicidad se define como su condición de ser únicos o irrepetibles en el área de estudio (repetitividad en el espacio). Fue, por ejemplo, uno de los criterios claves para la puesta en valor de los yacimientos de dinosaurios en la provincia argentina del Neuquén (Vejsbjerg et al., 2002) o para la declaración de Patrimonio de la Humanidad del Parque Nacional Talampaya y del Parque Provincial Ischigualasto en las provincias argentinas de La Rioja y San Juan, respectivamente ya que constituyen uno de los escasos ejemplos en el mundo de yacimientos del Triásico (Cortez et al., 2005). Unicidad y rareza son criterios con muchos puntos en común; la diferencia es que lo raro no tiene porque ser atractivo.

La buena conservación de los recursos es otra componente fundamental del atractivo de un recurso. Elementos destruidos, degradados de forma irreversible o alterada sustancialmente no podrán ponerse en valor recreativo. A este respecto hay que añadir que los recursos colindantes al atractivo deberán asimismo detentar un buen estado de conservación ya que constituyen el fondo escénico del mismo. La primera apreciación 
percibida por el observador-visitante integra todos los elementos del conjunto y quedará registrada con mucha fuerza en su imaginario. Por ello, es necesario que esta visión sea de calidad ya que de forma contraria costará mucho poner en valor el atractivo de forma aislada a su entorno.

Otro de los elementos de cierta relevancia en cuanto a los componentes de la atractividad es el tamaño o dimensiones del recurso. No existe una regla escrita al respecto, pero parece ser que los elementos de gran tamaño atraen más la atención que los pequeños aunque esta regla no es aplicable en el caso de cachorros o crías de animales. La base científica a este respecto podría radicar en las mejores condiciones de observación que tienen los recursos de gran tamaño, como es el caso de los árboles monumentales frente a plantas de diminuto tamaño, que aún siendo de gran interés o belleza no logran captar igualmente la atención del público menos especializado.

En lo que respecta a la emotividad, cabe señalar que todo aquel recurso con el que el visitante pueda generar una conexión emocional e intelectual basada en un concepto universal será más aprehensible y por tanto más atractivo (Ham, 1992). No siempre son evidentes estas conexiones $\mathrm{y}$, muchas veces, el cometido de desvelar los significados del recurso recaerá sobre el guía-intérprete ya que, en general, puede resultar muy complicado que un visitante de forma espontánea o intuitiva pueda dilucidarlo. Por eso resulta importante definir qué recursos son capaces por sí mismos de generar emociones y, por consiguiente, considerarse como los más atractivos de un sitio. Este es el caso de aquellos recursos que detentan una gran belleza escénica y en los que pueden incluirse tanto elementos abióticos como seres vivos. Aunque no existen demasiados estudios sobre las preferencias de los turistas por ciertas especies animales, existe una especial atención hacia los mamíferos, particularmente hacia especies emblemáticas (por ejemplo los grandes mamíferos de la sabana africana jirafas, elefantes, leones, etc., los cetáceos como las ballenas y delfines y la fauna de gran tamaño exótica como los osos pandas, canguros, koalas, etc.) así como hacia las aves (existe toda una industria alrededor del turismo ornitológico).

Otro criterio que contribuye a la valoración recreativa es la disponibilidad del recurso en el tiempo y en el espacio. Este criterio está relacionado con la capacidad de carga recreativa del ecosistema, las oportunidades para la visita, la frecuentación y la estacionalidad.

La capacidad de carga recreativa es una herramienta del análisis fundamental que no se puede obviar porque va a pautar el nivel óptimo de utilización del recurso o del ecosistema bajo condiciones de impacto mínimo. Existe numerosa literatura dedicada al tema desde diferentes metodologías de cálculo. Los autores de este trabajo incorporan en el análisis la componente del confort psicológico en un intento de garantizar al visitante una experiencia satisfactoria y de calidad en el lugar (Morant, 2007; Morant y Viñals, 2009).

La estacionalidad influye mucho en la valoración del lugar, en especial, en el caso de la fauna, cuya observación no siempre se puede garantizar, especialmente si se trata de especies migratorias como las aves o los cetáceos. Por otra parte, hay que señalar que el clima es determinante en las actividades de aire libre ya que pautará el periodo de disfrute del recurso. En este sentido, señalar que el clima deberá cumplir unos mínimos requerimientos de 
seguridad (baja incidencia de riesgos climáticos), de disfrute (máximo número de horas de insolación y mínimo número de días de lluvias en horario diurno) y de confort o bienestar para el cuerpo humano (umbrales térmicos y de humedad ambiental que no pongan en peligro el metabolismo humano) tal y como sugiere el método de Olgyay (1998).

La factibilidad se considera para valorar las repercusiones internas y externas de la opción turística. Tiene que ver con la viabilidad económica (evaluación de costes y beneficios, costes de adquisición de terrenos, costes de restauración del ecosistema, costes de gestión y mantenimiento, etc.), los beneficios sociales y la actitud de los actores locales (aceptación por parte de la comunidad local de las nuevas actividades propuestas). Se relaciona también con la existencia de derechos adquiridos (de paso, de uso, etc.), usos tradicionales del suelo (agrícolas, ganaderos, extractivos, etc.), prácticas religiosas (ritos, romerías, etc.) y capacidad de gestión de la institución que garantiza la protección de los recursos, entre otras.

La factibilidad está también relacionada con la idoneidad, es decir, con las características que tiene el lugar para el desarrollo de determinadas actividades recreativas. Así, por ejemplo, los deportes de invierno sólo se pueden desarrollar en áreas montañosas que cumplen unos mínimos requisitos en cuanto a espesor de nieve, pendientes, bajo riesgo de aludes, etc. Las actividades acuáticas como el rafting o la navegación en kayaks sólo se pueden desarrollar en cuerpos hídricos pero con fuertes corrientes, es decir en ríos de aguas bravas, mientras que el surf necesita de mares con ciertos parámetros de oleaje.

La accesibilidad se refiere a las facilidades de llegar hasta el ecosistema o lugar que se va a visitar. En parte existen unas dificultades de tipo natural como pendientes fuertes, intransitabilidad debido a la vegetación densa o a la presencia de lodos o terrenos encharcados, como ocurre en los humedales, pero también hay que considerar la dependencia de carreteras, caminos, equipamientos y servicios recreativos, señalización, transportes, etc. Este criterio determina el número de visitantes que accederán a ciertos atractivos. Se trata de un parámetro de obligado estudio porque una gran accesibilidad al espacio puede ser perjudicial ya que facilita la llegada masiva del público, lo cual es difícil de controlar por lo que puede impactar indiscriminadamente el ecosistema. Carreteras, ferrocarriles, cursos fluviales navegables y litorales accesibles generan oportunidades para el uso de los recursos pero también ocasionan contaminación y disturbios en general a los sistemas naturales (Gudinski et al., 2001). Por esto, cuanto mayor sea la accesibilidad a un espacio natural, mayor será su fragilidad. Así, por ejemplo, la densidad de carreteras en un espacio natural está en relación directa con los cambios en la composición de especies, incluyendo el incremento de especies exóticas invasoras y el descenso de la población de especies nativas, por mortalidad directa (atropellos, furtivismo, etc.), por modificaciones en los procesos hidrogeomorfológicos de los sistemas (cambios en las características físico-químicas de los suelos, etc.) y por fragmentación de los hábitats naturales, entre otros. Así, un sendero o ruta en un bosque templado modifica las condiciones microclimáticas que lo caracterizan (tasa de insolación, viento, etc.) y otras variables como el porcentaje de cubierta vegetal o el grado de compactación del suelo, entre otras. Asimismo, incrementa la fragmentación y crea una mayor densidad de borde que generalmente afecta negativamente la dinámica poblacional de muchas especies. 
Encontramos también una relación directa entre accesibilidad e incendios, los cuales, en función de su frecuencia e intensidad, pueden influir negativamente sobre la biodiversidad del paisaje (Burel y Baudry, 2002) y sobre sus elementos abióticos. Respecto a esto último, se ha demostrado que la estabilidad de los suelos es afectada por los incendios y por la compactación que supone el paso frecuente de personas y animales, lo cual disminuye la capacidad de infiltración con el incremento de las tasas de escorrentía superficial y por ende de las tasas de erosión en vertientes.

Finalmente hay que considerar el potencial que el ecosistema y los recursos tienen para el desarrollo de actividades de educación ambiental y/o para la interpretación del patrimonio. Los valores educacionales inherentes a algunos recursos son de gran importancia para fortalecer la sensibilización sobre la importancia de la conservación de los ecosistemas y para la transmisión de conocimientos relacionados con las ciencias naturales (ecología, biología, geología, hidrología, etc.) en el sentido de considerar los sitios naturales como "laboratorios vivos" para el aprendizaje.

El valor pedagógico de un lugar depende no sólo de las posibilidades del medio sino de la existencia de equipamientos (aulas de naturaleza, centros de interpretación, granjasescuela, senderos interpretativos, etc.), la calidad de los materiales educativos y el equipo de guías-intérpretes y monitores.

Respecto a la interpretación del patrimonio, hay que señalar que no todos los recursos presentan las mismas potencialidades. Los recursos más vistosos y atractivos suelen funcionar mejor en los programas de interpretación que aquellos que pasan más desapercibidos. Hay que señalar que un buen programa de interpretación temática con mensajes apropiados puede realzar en gran medida la atractividad de los recursos debido al valor añadido que comporta la introducción de componentes emocionales y las conexiones intelectuales como anteriormente se ha esbozado.

\section{DISCUSION Y CONCLUSIONES}

Cada uno de los criterios presentados en este trabajo tiene importancia por sí mismo, con lo cual su análisis y evaluación debe ser individualizada. Puede darse el caso que un sitio sea considerado como resistente respecto a varios de sus parámetros pero muy vulnerable a cierta actividad o impacto provocado por los visitantes. En ese caso, las medidas correctoras ante una acción que se quiera llevar a cabo deberían estar enfocadas sobre ese criterio en particular, a fin de mitigar los efectos sobre los componentes estructurales y/o funcionales que tengan relación con él. En cualquier caso, se debe hacer una evaluación global que deberá ser el producto de un análisis integrado y una reflexión sobre los "pros" y "contras" de los resultados alcanzados.

Queda claro que, en varios de los criterios propuestos, resulta necesario tener una información muy completa sobre el ecosistema que no siempre está disponible. En relación a ello, y mientras se adquieran los datos necesarios, el gestor deberá tomar decisiones de acuerdo al nivel de conocimiento en ese momento del espacio que administra, aún cuando 
tenga que evaluar parcialmente alguno de los criterios en función de la información disponible. En esos casos, el gestor deberá recordar el principio precautorio que plantea que la falta de información debe ser ponderada en la evaluación, en función de la existencia de un posible componente desconocido de fragilidad en el sistema.

Por otra parte, hay que tener presente que todas las acciones llevadas a cabo en un sistema natural tendrán en mayor o menor medida algún tipo de impacto sobre el medio. Por lo tanto, si por esta evaluación un gestor llega a la conclusión de que un espacio natural es poco frágil, esto no implica que se pueda realizar allí cualquier tipo de actividad y con cualquier intensidad. Los resultados de la aplicación de este tipo de valoración van a permitir, por una parte identificar con antelación los posibles impactos que la actividad causará y así planificar desde el principio medidas preventivas y, por otra parte, determinar la vocación de los recursos para determinadas actividades recreativas.

Finalmente, cabe recordar que la valoración intrínseca de los recursos no siempre coincide con la valoración recreativa ya que en ésta última, como hemos visto, influyen componentes subjetivos y factores externos no inherentes a los elementos del ecosistema.

\section{REFERENCIAS BIBLIOGRÁFICAS}

BACKMAN, S.J., MUZAFFER, U. Y BACKMAN, K. (1991). Regional analysis of tourism resources. Annals of Tourism Research, 18 (2): 323-328.

BURKART, J. Y MEDLINK, C. (1986). Tourism, past, present, and future. Oxford: Heinemman, London.

BUREL, F. Y BAUDRY, J. (2002). Ecología del paisaje: Conceptos, métodos y aplicaciones, Madrid, Mundi-Prensa.

CADIÑANOS, J.A. Y MEAZA, G. (1998). Bases para una biogeografía aplicada: criterios y sistemas de valoración de la vegetación, Logroño, Geoforma Ediciones.

CORTEZ, E., GIANNONI, S.M. Y BORGHI, C.E. (2005). Plan de manejo del Parque Provincial Ischigualsto, Pcia. de San Juan, Período 2005-2010. Fase I. Informe Técnico, 146 pp.

DE ANGELIS, D.L., BARTELL, S.M. Y BRENKERT, A.L. (1989). Effects of nutrient recycling and food-chain length on resilience. The American Naturalist 134:5, pp.778-805.

DEFERT, P. (1960). Le tourisme dans l'expansión regionale. Principes et methods. AIEST, Vol. I. Editions Gurten, Berna.

GUDINWKI, H., FURNISS, M.J., ZIEMER, R.R. Y BROOKES, M.H. (2001). Forest Roads: A Synthesis of Scientific Information. Portald (OR): USDA Forest Service, Pacific Northwest Research Station, General Tehcnical Report PNW-GTR-509.

GUNN, C.A. (1988). Vacationscapes: Designing tourist regions. Van Nostrand Reheinhold, New York.

HAM, S.H. (1992). Environmental Interpretation. A practical guide for people with big ideas and small budgets. Ed. Fulcrum Publishing, $456 \mathrm{pp}$.

IUCN (2000). Global Invasive Species Database. 100 of the World's worst invasive species. Invasive Species Specialist Group (GISP). www.issg.org/database/search.aps?st=100ss\&fr 
IUCN (2001). The IUCN Red List of threatened species. 2001 Categories \& Criteria. http://www.iucn.org/themes/ssc/redlists/RLcats2001booklet.html

KARR, J.R. (1996). Ecological integrity and ecological health are not the same, en P.C. Schulze (ed.), Engineering within ecological constraints, Washington DC, National Academic Press, pp. 97-109.

LENO, F., (1993). Técnicas de evaluación del potencial turístico. Serie Libros sobre Turismo, no 2, Ministerio de Industria, Comercio y Turismo, MICYT, Madrid, $261 \mathrm{pp}$.

LIDDLE, M. (1997): Recreation Ecology. The ecological impact of outdoor recreation and ecotourism, London, Chapman \& Hall.

MALLARACH, J.M. (1999). Criteris i mètodes d'avaluació del patrimoni natural. Documents dels Quaderns de medi ambient №2, Barcelona, Departament del Medi Ambient de la Generalitat de Catalunya. 2:1-106.

MEFFE, G.K. Y CARROLL, C.R. (1997). The species in conservation. En Meffe y Carroll (eds.): Principles of conservation biology ( $2^{\text {nd }}$ Ed.), Sunderland, Sinauer Associates, pp. 57-86.

MORANT, M. (2007). Desarrollo de un modelo para la determinación de la capacidad de carga recreativa y su aplicación espacios naturales protegidos de la Comunidad Valenciana. Tesis Doctoral. Valencia, Universidad Politécnica de Valencia.

MORANT, M. Y VIÑALS, M.J. (2009). Modelo para evaluar la capacidad de carga recreativa en áreas de uso intensivo de espacios protegidos. Casos de estudio de la Comunidad Valenciana (España). En López Olivares (ed.): Turismo y gestión de espacios protegidos, Ed. Tirant lo Blanch, pp. 618-636.

NAEEM, S. (1998). Species redundancy and ecosystem reliability, Conservation Biology 12, pp.39-45.

NUSSER, S.M. Y GOEBEL, J.J. (1997): The National Resources Inventory: a long-term multiresource monitoring programme, Environmental and Ecological Statistics. Springer Netherlands. Vol. 4, Number 3, pp. 181-204.

OLGYAY, V. (1998): Arquitectura y Clima. Manual de diseño bioclimático para arquitectos y urbanistas. Ed. Gustavo Gili.

ORGANIZACIÓN de los ESTADOS AMERICANOS (OEA) (1978). Metodología de inventario turístico, mimeografiado, Washington.

ORGANIZACIÓN MUNDIAL DEL TURISMO (OMT) (1978). Evaluación de los recursos turísticos, O.M.T. Madrid.

RABINOWITZ, D. (1981). Seven forms of rarity. En: Synge (ed.): The biological aspects of rare plant conservation. Ed. John Wiley, Chichester, UK. Pp. 205-217

STRICKLAND-MUNROA, J., ALLISON, H. Y MOORE, S.(2010). Using resilience concepts to investigate the impacts of protected area tourism on communities. Annals of Tourism Research, 37 (2): 499-519.

VEJSBJERG, L., CALVO, J.O. Y HEREDIA, S. (2002). Criterios de valoración turística -recreativa de recursos geológicos, paleontológicos y paisajísticos. Caso Monumento Natural Parque de los Dinosaurios. Actas del XV Congreso Geología Argentino, Ciudad de Neuquén.

VIÑALS, M.J. (1999). Los espacios naturales y rurales. Nuevos escenarios del turismo sostenible. En: Viñals y Bernabé (coords.): Turismo en espacios naturales y rurales. Universidad Politécnica de Valencia, pp. 13-34. 
VIÑALS, M.J., FILIBERTO, I. Y MORANT, M. (2005). Manual para o inventário dos valores culturais das Zonas Húmidas / Manual para el inventario de los valores culturales de los humedales. Ed. Instituto da Conservaçao da Natureza, Ministério do Ambiente (Portugal), 179 pp. 